\title{
SIMHYDRO: collective intelligence session "MOCCAFAI: towards a new generation of hydraulics models: smart, collaborative"
}

\author{
Jean-Michel TANGUY ${ }^{1}$, Cicely PAMS-CAPOCCIONI ${ }^{2}$, Anna DUPONT ${ }^{1}$
}

\author{
${ }^{1}$ SHF - 25 rue des Favorites, 75015 Paris, jm.tanguy@shf-hydro.org \\ ${ }^{2} S N C F$
}

\begin{abstract}
A collective intelligent session was offered to participants at SIMHYDRO conference 2017 to address in an innovative way the issues pertaining to simulation in hydraulics using the latest new technologies. 20 participants divided into 3 groups designed 3 innovative projects on field data collection, processing and dissemination services. This could be the kick-off of a start-up...

RÉSUMÉ. - un séminaire d'intelligence collective a été proposé aux participants de la conférence SIMHYDRO 2017. Il consistait à repenser le monde de la simulation hydraulique. 20 participants répartis en 3 groupes ont proposé 3 projets innovants dans le domaine du service de données terrain : collecte, traitement et diffusion. Ils pourraient constituer le démarrage d'un projet de start-up...
\end{abstract}

\section{THE CONTEXT OF SIMULATION}

Simulation tools are common nowadays in many hydraulics scientific disciplines. They allow for the replication of nature and its behavior, and its interactions with materials and man-made constructions in rivers, estuaries and coasts.

The real start of the development of these tools was in the 1970's with the development of informatics which created a change of paradigm. The new capacities of computers, the possibility to visualize inputs and results allowed research teams to develop new simulation tools, which superseded analytical formulas and analogic machines.

Significant developments of various simulation tools were then applied to bigger physical domains with better accuracy, shorter time steps and longer periods. Processes' interactions using the coupling of physical processes became possible and hydrodynamics and sedimentology with bed evolutions in fluvial and maritime environments.

Initially developed in the car industry, real time mesh conception used for designing cars gave rise in the 80 's to increasingly user-friendly (pre and post treatments) representation of the reality.

In our scope of interest (free surface flow simulation, including river, estuarine, maritime environments), numerical tools have improved, running faster and more accurately, becoming more user-friendly thereby offering better and ever-more precise tools to decision-makers.

Yet, over the last decade, models have benefited mainly from optimization improvements and less so from fundamental evolutions: numerical schemes have been optimize, pre and post-treatments of data have allowed for the representation of fast computed evolution processes like floods, storm surges...

In a world that changes drastically however, the emergence of new technologies provides us with a new perception of our environment. One may therefore ask the following questions:

- Is our scientific community taking advantage of the latest technological evolutions?

- Is our scientific community taking into account the changes in behavior and mind set of the new generation?

- Is our scientific community effectively meeting today's social needs?

Looking at other fields in engineering sciences, one may identify some strategic innovations:

- WAZE: a crowdsourcing, collaborative app which keeps the driver informed of traffic jams using the geographic positioning of hundreds and hundreds of drivers and offering new itineraries. This type of app adds-on or encapsulates the traditional traffic forecasting models.

- WATSON: an IBM cognitive technology platform, which after defeating Kasparoff chess world champion, solves non algorithmic problems in various domains: health, engineering, big data. WATSON can be directly connected to the web.

- IPV6: the new Internet protocol gives all the connected objects an ID which could give its position: a bath or a caravan drifting on a flooding river could now act as a sensor...

- «big data » and the associated processing tools (data mining) may offer a precise knowledge of what happens in a flooded area

- Huge sets of new heterogeneous data: tweets, photos, videos produced by people may be integrated as field measurements in simulation tools. The pro-active behavior of people impacted by a natural event (residents, tourists, public security professionals...) may produce data and thereby increase the knowledge on a disaster's as it evolves in time

- Today, with SPH models, it is possible to interact in real time using modeling tools, and to change hydraulic conditions as well as the topography, even though this is still applicable to only small domains, especially in industrial fluid mechanics.

\section{THE COLLABORATIVE SESSION}

On the basis of this background, the 20 participants who joined the collective intelligence session were invited to think differently, in an innovative and creative way in order to establish new approaches or new products in the field of hydraulics.

The exercise was for each of the 3 groups to aim at launching by the end of the session a start-up project with an innovative product including a business model.

So as to be able to come up with groundbreaking ideas and projects, today's current practices in engineering were presented and illustrated using 4 case-studies of simulation (Aude 1999, Marseilles 2002, Saumane 2012, Vietnam 2017). All cases were using data bases, pretreatment, simulation results on dense meshes and post treatment ready to give the decision makers the best representation of flood situations and consequences on the ground.

The most strategic point was that these results did not integrate so much the new technologies nor did they include in any way social behavior. It was mentioned that in a former workshop organized by the French Hydro Society (SHF) last May, 3 groups were invited to reflect on their needs regarding modeling tools: university labs, engineering offices and decision makers. These groups came up with the following items:

- When results of simulation tools are presented to decision makers, they would like to " play » with the model themselves, understand its sensitivity, integrate new construction works, featuring dikes decays, dam gates opening... They want to play with interactive modeling, as with real water in physical models, so as to increase their understandings.

- All types of field data have to be integrated in real-time running models so as to take into account the evolution of the reality.

- New thinking has to come about, allowing for the making of open collaborative exchange platform, in which all type of modeling customers can improve and run the simulation tools

This is why it appears that the new simulation tool generation has to change drastically.

\section{THE RESULTS}

At Simhydro 2017, the 3 groups worked during a half-day session and proposed 3 projects:

- RISK-HUB which focuses on collection, treatment and dissemination of every type of field data (sensor devices, drone or satellite images, photos, videos...). The producers (individuals, private or public services) can be the end-users through a collaborative data center. The service type activity is dedicated to end-users which can be charged to users.

- COPT'EAU is a platform for field data which can gather and provide raw data as well as enriched data in real time during a crisis event designed for each customer. This B to B service provides data to forecasting services, public security services, municipalities... The service can be charged to users.

- BET BIG is a new service based on collecting the huge amount of data produced by all the sensors due to the extension of connected objects and the development of sensors everywhere: cars, boats, smartphones. The customers will be academic staff, insurance companies, utilities as well as engineering companies.

This exercise of collaborative creativity is proof of a sound interest from the community to invest in redefining new approaches and new concepts using the new technologies for the benefit of people.

The next step will be to set up a think-tank on this approach led by SHF and involving all interested parties.

To join this group, please send your coordinates to jm.tanguy@ shf-hydro.org 


\section{Comité d'organisation :}

\section{Animateur du colloque :}

Jean-Michel Grésillon (MEEM-SHF)

- $\quad$ Stéphanie Bidault (CEPRI)

- $\quad$ Philippe Bolo (ISL)

- $\quad$ Anne Chanal (Cerema)

- $\quad$ Thierry Coanus (ENTPE)

- $\quad$ Anna Dupont (SHF)

- $\quad$ Michel Lang (Irstea)

- $\quad$ Daniel Loudière (SHF)

- $\quad$ Didier Richard (Irstea)

- $\quad$ Neda Sheibani (SHF)

- Dominique Thierry (MEEM)

- $\quad$ Fredy Vinet (Univ.Montpellier III)

\section{Dates importantes :}

Fin mai 2016:

Appel à communications

- 0 décembre 20 | 6: date limite de la réception des résumés

- 17 mars 2017 : Notification aux

auteurs

- I5 mai 2017 : Envoi des textes complets.

n.sheibani@shf-hydro.org
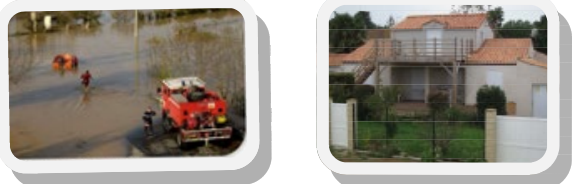

A l'occasion de la présentation des résultats des travaux de recherche conduits dans le cadre du programme de recherche " Risques, Décision, Territoires » du MEEM sur le thème de « La résilience des territoires face aux risques », la SHF s'associe au MEEM pour organiser un colloque sur les « apports de la notion de résilience à la gestion des risques ».

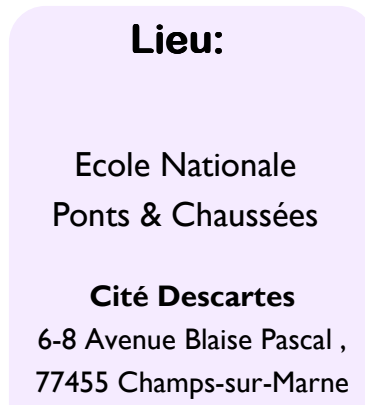

Risques et Résilience des Territoires 2017

Apports de la notion de résilience à la gestion des risques
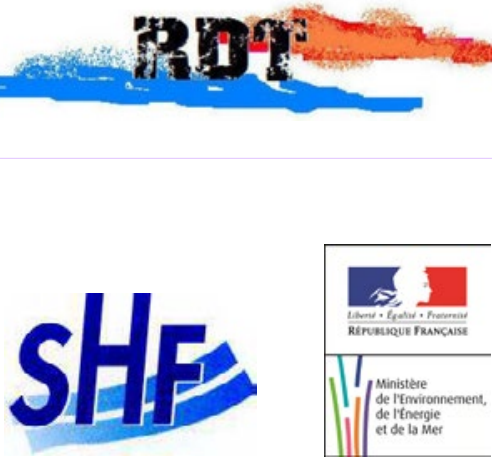

10 - 11 octobre 2017

ENPC - Marne La Vallée

\section{LCWI Laboratoire de machines hydrauliques}

Un laboratoire de l'EPFL spécialisé dans I'hydrodynamique des turbines, des pompes, des pompes-turbines et des installations hydrauliques.

Expertise scientifique de plus de 40 ans au service de l'industrie des machines hydrauliques et du secteur électrique : analyse d'écoulements, cavitation, hydro-acoustique et instrumentation avancée.

Evaluations sur site du rendement, du risque de cavitation et du comportement hydro-acoustique des installations hydrauliques.

Validations expérimentales, suivant la norme CEI 60193, des performances sur modèle réduit des machines hydrauliques et vannes destinées aux centrales hydro-électriques en cours de réalisation ou en rénovation.

Actions de formation : Master en génie mécanique, doctorat, cours annuel de spécialisation "machines hydrauliques", autres cours et possibilités de stages sur demande.

EPFL - LMH

Avenue de Cour 33bis $\mathrm{CH}-1007$ LAUSANNE Suisse

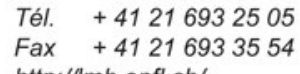

http://lmh.epfl.ch/

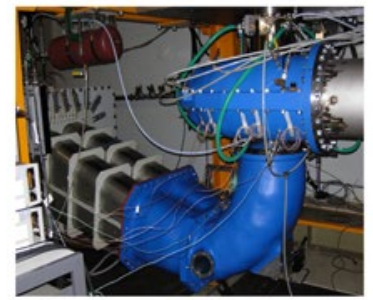



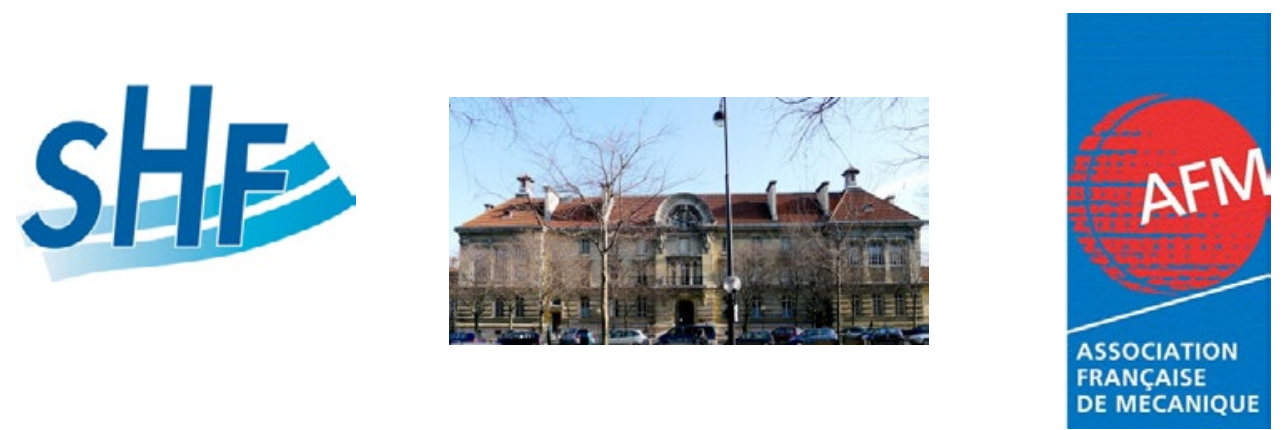

\author{
Journée s S H F / A F M : \\ Machines Hydrauliques et Cavitation/ \\ Hydraulic Machines and Cavitation \\ 8 et 9 Novembre 2017, Paris, France \\ November 8-9 $9^{\text {th }}, 2017$, Paris, France
}

Les prochaines journées SHF/AFM sur les machines hydrauliques et la cavitation se tiendront les 8 et 9 Novembre 2017 sur le site du Campus ENSAM de Paris. Les chercheurs et industriels travaillant dans ces domaines sont invités à présenter leurs travaux et soumettre au préalable un résumé d'une page avant le 8 Septembre 2017. La conférence couvrira les aspects théoriques, expérimentaux et numériques et offrira une plateforme pour des échanges scientifiques et retours d'expériences.

The next SHF-AFM conference on Hydraulic Machines and Cavitation is scheduled on November the $8^{\text {th }}$ and $9^{\text {th }}, 2017$ in Paris ENSAM Campus. Researchers and industrials working in these areas are invited to present their works and submit an abstract before September $8^{\text {th }}, 2017$. The conference will address theoretical, experimental and numerical aspects and will offer a platform for scientific exchange.

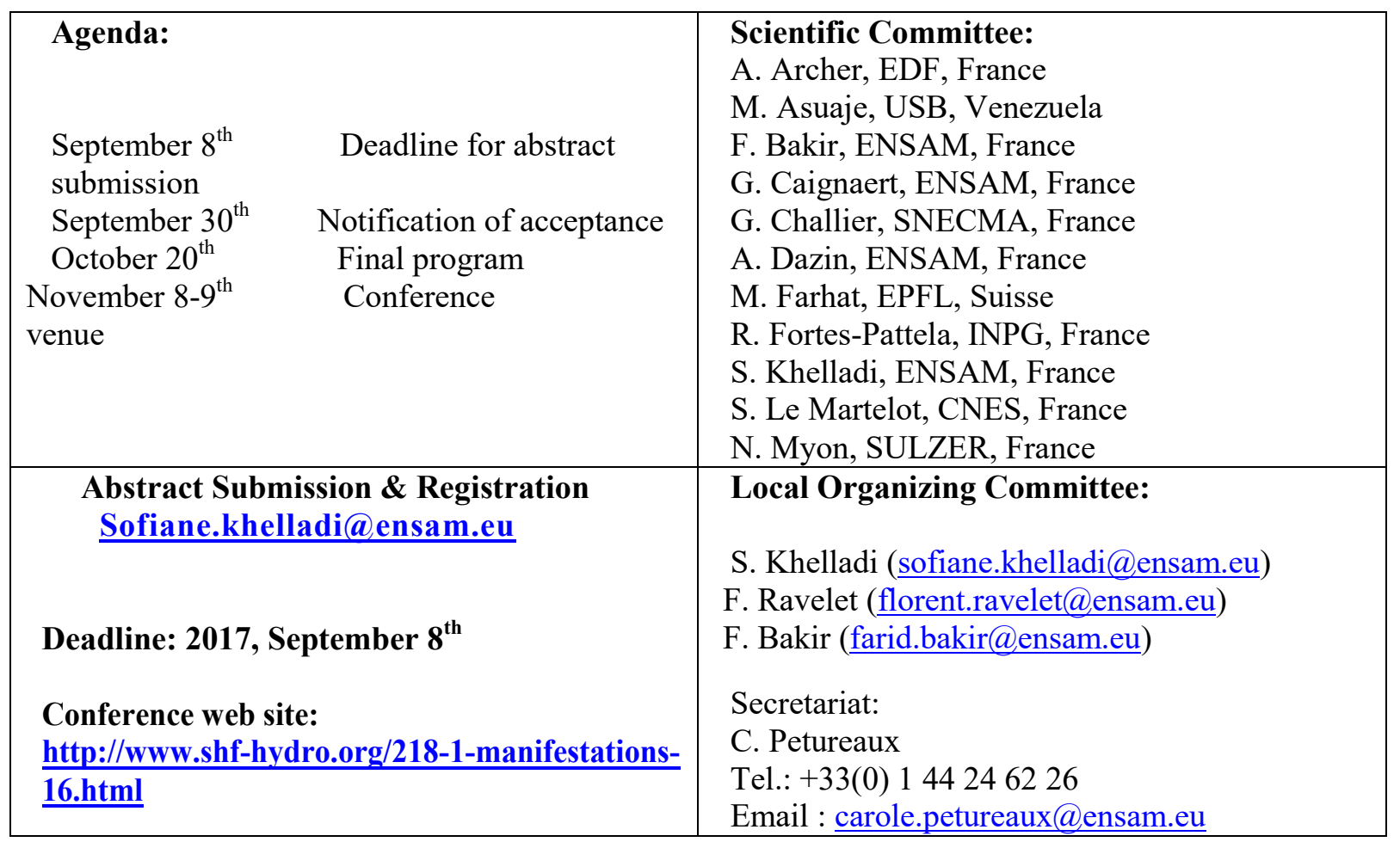




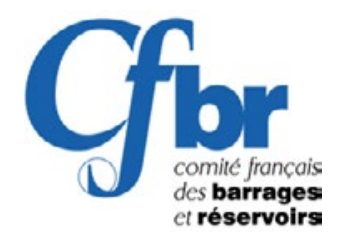

http://www.barrages-cfbr.eu/

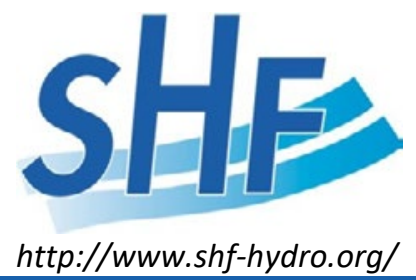

\section{Colloque \\ Hydraulique des barrages et des digues}

\section{9 et 30 novembre 2017, Chambéry}

Attention au changement de date

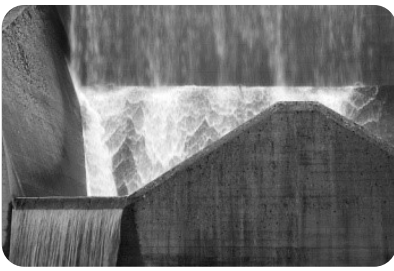

Barrage de Corscia (C)BETCGB - Stéphan AIGOUY

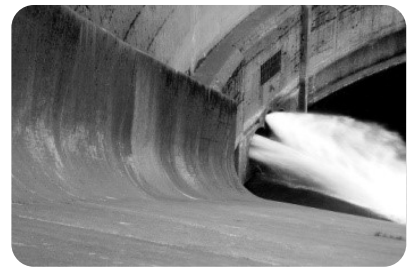

Barrage de Palisse (C) BETCGB

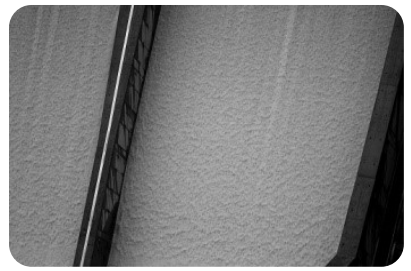

Barrage de Rizzanèse (C)BETCGB - Stéphan AIGOUY

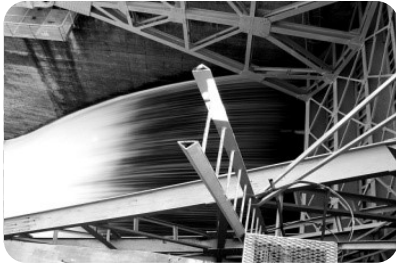

Barrage de Yaté (C)BETCGB - Stéphan AIGOUY

\section{$2^{\mathrm{e}}$ ANNONCE}

Le Comité Français des Barrages et Réservoirs (CFBR) et la Société Hydrotechnique de France (SHF) organisent régulièrement des colloques qui visent à rassembler les administrations, les maîtres d'ouvrages et exploitants d'ouvrages hydrauliques, les ingénieursconseils, les entrepreneurs, les chercheurs et enseignants ainsi que les experts individuels.

\section{En France, le contexte des ouvrages hydrauliques a été marqué récemment par :}

- l'évolution de la législation et de la réglementation en matière de sécurité des ouvrages, avec le " décret du 12 mai 2015 relatif aux règles applicables aux ouvrages construits ou aménagés en vue de prévenir les inondations et aux règles de sûreté des ouvrages hydrauliques » et le projet d" "arrêté fixant des prescriptions techniques relatives à la sécurité des barrages";

- de nombreux projets, à l'étude ou réalisés, de modification substantielle ou de révision spéciale de barrages ou de digues concernant des composants hydrauliques des ouvrages ;

- des développements majeurs relatifs à la conception des déversoirs en touches de piano (piano key weirs) qui ont été exposés lors de deux séminaires spécifiques (PKW 2011, PKW 2013);

- des conceptions nouvelles (digues fusibles, marches d'escalier, ...) pour lesquelles le retour d'expérience des ouvrages réalisés devient appréciable.

Le retour d'expérience du comportement des ouvrages hydrauliques des barrages et des digues en fonctionnement, notamment en cas d'incident (une majorité des ruptures de barrages étant due aux évacuateurs de crues), et leur suivi constituent des bases essentielles pour nos pratiques. La gestion en temps réel des ouvrages lors des crues et l'application des consignes correspondantes (y compris lors de travaux) sont aussi des préoccupations majeures. Sont également à considérer l'apport des études de dangers et l'amélioration de nos connaissances des organes traditionnels (vannes, clapets, bassins de dissipation, ...) sous sollicitations particulières (surcotes hydrauliques, embâcles, séismes, ...).

Le colloque organisé les 29 et $\mathbf{3 0}$ novembre 2017 par le CFBR et la SHF sera donc consacré à l'hydraulique des barrages et des digues.

Ce colloque permettra de réviser de façon approfondie le fonctionnement des composants hydrauliques des barrages et des digues, que ce soient les moyens d'études, la conception, la réalisation ou le comportement en situation réelle. Ceci concerne tous les dispositifs hydrauliques, en priorité les évacuateurs de crue mais également les ouvrages de prise, de vidange, de dérivation provisoire (en cours de travaux) et de gestion du réservoir.

Les principaux thèmes sont provisoirement répartis comme suit :

i. méthodes de conception des ouvrages hydrauliques des aménagements et méthodes de justification en situations normales et extrêmes : méthodes analytiques, modèles physiques, modèles numériques, ... ;

ii. retour d'expérience des ouvrages réalisés : modes de construction, de confortement ou de réparation, comportement en cas de forts débits, adéquation avec le projet initial ;

iii. gestion des ouvrages en période de crue et des sollicitations particulières : embâcles, glaces, avalanches, chutes de blocs, entraînement d'air, ... ;

iv. apport des études de dangers, qualification des scénarios défavorables, probabilités associées, évolutions attendues, perception de la sécurité hydraulique, points de vue des acteurs, notamment des services de contrôle. 


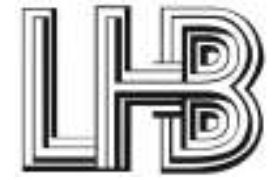

$2007 \quad N^{\circ} 1$ et $2 \quad$ Anniversaire des grandes crues de 1856

$\mathrm{N}^{\circ} 3$ Hydroinformatique

$\mathrm{N}^{\circ} 4$ et $5 \quad$ Qualité des eaux marines, Hydrologie urbaine

$N^{\circ} 6 \quad$ Variations climatiques et hydrologie (1), Microfluidique

$2008 \quad N^{\circ} 1 \quad$ Risques littoraux majeurs

$\mathrm{N}^{\circ} 2 \quad$ Variations climatiques et hydrologie (2)

$N^{\circ} 3$ et $5 \quad$ Gestion active des eaux

$N^{\circ} 4$ et $6 \quad$ Gestion sociale et économique de l'eau

$\mathrm{N}^{\circ} 4$ et $5 \quad$ Transports solides et gestion des sédiments

$2009 \quad N^{\circ} 1 \quad$ Transports solides et gestion des sédiments

$N^{\circ} 1$ et 2 Risques côtiers

$N^{\circ} 3$ et $4 \quad$ Mesures Hydrologiques et incertitudes

$\mathrm{N}^{\circ} 4 \quad$ Ecoulements diphasiques bouillants

$\mathrm{N}^{\circ} 5$ et 6 Prévisions hydrométéorologiques, Hydrologie urbaine et Microfluidique

$2010 \quad N^{\circ} 1$ et 2

$\mathrm{N}^{\circ} 1$ et 2

$\mathrm{N}^{\circ} 2$ et 3

$\mathrm{N}^{\circ} 4$ et 5

$\mathrm{N}^{\circ} 6$

$\mathrm{N}^{\circ} 6$

$\mathrm{N}^{\circ} 6$

$2011 \quad N^{\circ} 1$ et 2

$\mathrm{N}^{\circ} 2$

$\mathrm{N}^{\circ} 3$

$\mathrm{N}^{\circ} 4$

$\mathrm{N}^{\circ} 5$

$\mathrm{N}^{\circ} 6$

$2012 \quad \mathrm{~N}^{\circ} 1$ et 2

$\mathrm{N}^{\circ} 2$

$\mathrm{N}^{\circ} 3$

$\mathrm{N}^{\circ} 4-5$

$\mathrm{N}^{\circ} 6$

$2013 \quad N^{\circ} 1$

$\mathrm{N}^{\circ} 2$

No3

$\mathrm{N}^{\circ} 4$

$\mathrm{N}^{\circ} 5$

$\mathrm{N}^{\circ} 6$

$2014 \quad N^{\circ} 1$

$\mathrm{N}^{\circ} 2,3,4,5$

$\mathrm{N}^{\circ} 3,4,5$

$\mathrm{N}^{\circ} 6$

2015

$\mathrm{N}^{\circ} 1,2,3,4$

$\mathrm{N}^{\circ} 2,4$

$N^{\circ} 1,2,3$,

4,5

$\mathrm{N}^{\circ} 4,5,6$

$\mathrm{N}^{\circ} 5,6$

2016

$\mathrm{N}^{\circ} 1,2,3,4$

$N^{\circ} 1,6$

$\mathrm{N}^{\circ} 2,3,4,5$

$\mathrm{N}^{\circ} 4,5,6$

$2017 \quad N^{\circ} 1$

Evacuateurs de crue

Hydrologie urbaine

Imagerie satellite

Etiages sécheresses canicules

Sédimentation côtière

Modèles physiques hydrauliques

Cavitation

Risque inondation en lle de France

Prévisions hydrologiques

Hydraulique

Hydrodynamique, Hydrologie urbaine et Microfluidique

Niveaux marins, Séries pluvio anciennes, dynamique nivale, Hydrologie urbaine

Environnement et hydroélectricité, Modélisation hydraulique, Houle en estuaires

Eau en montagne

Environnement et hydroélectricité

Stockage d'énergie par pompage, Cavitation, Hydrométrie

Stockage d'énergie par pompage, Erosion des ouvrages, Eau et énergie

Protection contre les inondations, Dérive littorale, Erosion

Risque inondation, Affouillement de barrage, Ecoulement en canal

Modèles hydrauliques, Crues, Microfluidique

Crues historiques, Erosion, Mécanique des fluides

Assainissement, Vulnérabilité face aux inondations, Mesure des MES, Microfluidique

Hydrologie de montagne, Microfluidique, Assainissement

Hydrométrie et incertitudes

Réseaux de mesure, Evénements extrêmes fluviaux et côtiers

Evénements extrêmes d'inondation

Hydrométrie

GIS Hydraulique pour l'Environnement et le Développement Durable

Energies marines,

Modélisation des écoulements, simulation

Risques Inondations, événements hydrologiques extrêmes ; Hydrologie urbaine

Rénovation des barrages

Simulation hydraulique

Drones et hydraulique

Barrages

Risque inondation

Climat et ressources en eau

Stockage d'énergie, Hydroélectricité et environnement, Hydrodynamique,

Ressources en eau

Commande auprès de La Houille Blanche - SHF - 25 rue des Favorites, F 75015 PARIS

Tél. +33 (0)142509103 - Courriel lhb@shf-hydro.org - www.shf-hydro.org

\begin{tabular}{c}
\hline Prix TTC : \\
\hline$\square$ France : 20 euros + port \\
$\square$ Etranger : 24 euros + port \\
\hline
\end{tabular}




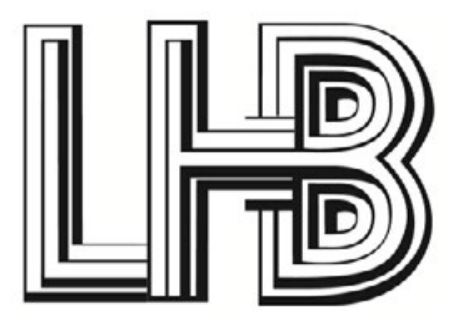

LA HOUILLE BLANCHE

\section{Revue internationale de leau}

L'eau ressource et danger, ses aménagements, ses usages pour l'homme et son environnement : de la mécanique des fluides aux aménagements hydrauliques et aux risques naturels.

Revue avec Comité de lecture, référencée dans les grandes bases internationales

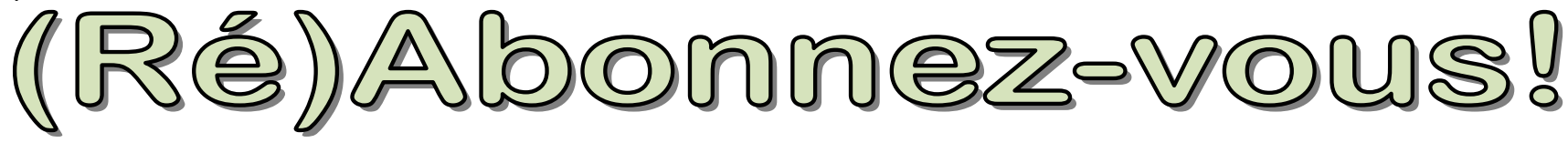

6 numéros par an - disponible en ligne

Tous les articles parus depuis 1902, soit toute l'histoire de l'hydraulique moderne, sont disponibles en ligne.

\section{www.shf-Ihb.org}

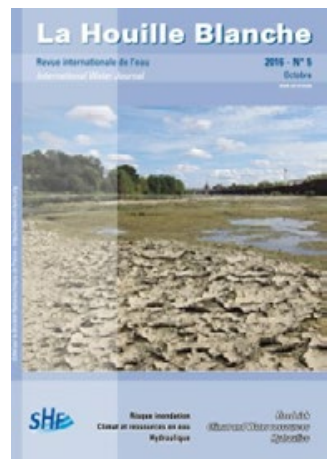

Editeur :

SOCIETE HYDROTECHNIQUE DE FRANCE (SHF)

25 rue des Favorites - F75015 - Paris

Tél. 33 (1) 42509103 - Fax 33 (1) 42505983

Courriel.Ihb@shf-hydro.org

Code TVA FR 23784309056

\section{Bulletin d'abonnement à retourner à}

\section{La Houille Blanche - 25 rue des Favorites - F75015 PARIS .}

Nom/Name :

Société/Company :

Prénom/First Name

Adresse/Address :

Code postal/Postal code :

Pays/Country:

Mail : Ville/City

Tél/Phone :

Fonction/Function:

Fax :

\section{Tarifs 2017 pour les abonnements individuels}

Les 6 numéros TTC de (Euros) (TVA $2.10 \%$ incluse)

Edition papier seul

France : 139

Etranger : 174
Edition électronique seule

139
Edition papier + électronique

France : 172

Etranger : 204

Nous consulter pour les sociétés

Tarifs spéciaux pour les adhérents SHF et AIRH 